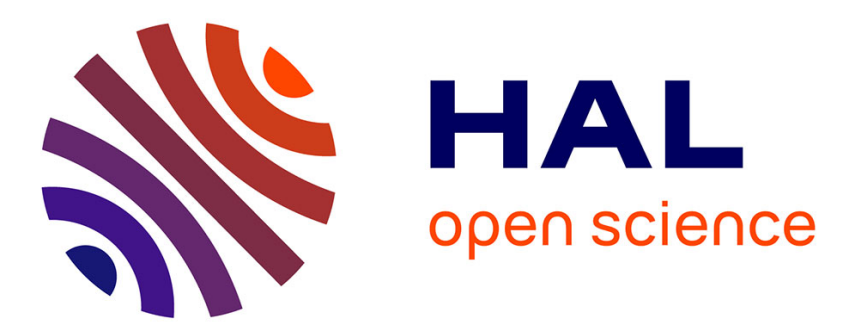

\title{
Efficient Möbius Transformations and their applications to D-S Theory
}

Maxime Chaveroche, Franck Davoine, Véronique Cherfaoui

\section{To cite this version:}

Maxime Chaveroche, Franck Davoine, Véronique Cherfaoui. Efficient Möbius Transformations and their applications to D-S Theory. 13th International Conference on Scalable Uncertainty Management (SUM 2019), Dec 2019, Compiègne, France. pp.390-403, 10.1007/978-3-030-35514-2_29 . hal02326438

\section{HAL Id: hal-02326438 \\ https://hal.science/hal-02326438}

Submitted on 22 Oct 2019

HAL is a multi-disciplinary open access archive for the deposit and dissemination of scientific research documents, whether they are published or not. The documents may come from teaching and research institutions in France or abroad, or from public or private research centers.
L'archive ouverte pluridisciplinaire HAL, est destinée au dépôt et à la diffusion de documents scientifiques de niveau recherche, publiés ou non, émanant des établissements d'enseignement et de recherche français ou étrangers, des laboratoires publics ou privés. 


\title{
Efficient Möbius Transformations and their applications to D-S Theory ${ }^{\star}$
}

\author{
Maxime Chaveroche $\mathrm{e}^{1[0000-0002-0834-4022]}$, Franck \\ Davoine $^{1[0000-0002-8587-6997]}$, and Véronique Cherfaoui ${ }^{1}$ \\ ${ }^{1}$ Alliance Sorbonne Université, Université de Technologie de Compiègne, CNRS, \\ Laboratoire Heudiasyc \\ 57 Avenue de Landshut, 60200 Compiègne, France, name.surname@hds.utc.fr
}

\begin{abstract}
Dempster-Shafer Theory (DST) generalizes Bayesian probability theory, offering useful additional information, but suffers from a high computational burden. A lot of work has been done to reduce the complexity of computations used in information fusion with Dempster's rule. The main approaches exploit either the structure of Boolean lattices or the information contained in belief sources. Each has its merits depending on the situation. In this paper, we propose sequences of graphs for the computation of the zeta and Möbius transformations that optimally exploit both the structure of distributive lattices and the information contained in belief sources. We call them the Efficient Möbius Transformations (EMT). We show that the complexity of the EMT is always inferior to the complexity of algorithms that consider the whole lattice, such as the Fast Möbius Transform (FMT) for all DST transformations. We then explain how to use them to fuse two belief sources. More generally, our EMTs apply to any function in any finite distributive lattice, focusing on a meet-closed or join-closed subset.
\end{abstract}

Keywords: zeta transform, Möbius transform, distributive lattice, meetclosed subset, join-closed subset, Fast Möbius Transform, FMT, DempsterShafer Theory, DST, belief functions, efficiency, information-based, complexity reduction.

\section{Introduction}

Dempster-Shafer Theory (DST) [11] is an elegant formalism that generalizes Bayesian probability theory. It is more expressive by giving the possibility for a source to represent its belief in the state of a variable not only by assigning credit directly to a possible state (strong evidence) but also by assigning credit to any subset (weaker evidence) of the set $\Omega$ of all possible states. This assignment of credit is called a mass function and provides meta-information to quantify

\footnotetext{
* This work was carried out and co-funded in the framework of the Labex MS2T and the Hauts-de-France region of France. It was supported by the French Government, through the program "Investments for the future" managed by the National Agency for Research (Reference ANR-11-IDEX-0004-02).
} 
the level of uncertainty about one's believes considering the way one established them, which is critical for decision making.

Nevertheless, this information comes with a cost: considering $2^{|\Omega|}$ potential values instead of only $|\Omega|$ can lead to computationally and spatially expensive algorithms. They can become difficult to use for more than a dozen possible states (e.g. 20 states in $\Omega$ generate more than a million subsets), although we may need to consider large frames of discernment (e.g. for classification or identification). Moreover, these algorithms not being tractable anymore beyond a few dozen states means their performances greatly degrade before that, which further limits their application to real-time applications. To tackle this issue, a lot of work has been done to reduce the complexity of transformations used to combine belief sources with Dempster's rule [6]. We distinguish between two approaches that we call powerset-based and evidence-based.

The powerset-based approach concerns all algorithms based on the structure of the powerset $2^{\Omega}$ of the frame of discernment $\Omega$. They have a complexity dependent on $|\Omega|$. Early works $[1,7,13,12]$ proposed optimizations by restricting the structure of evidence to only singletons and their negation, which greatly restrains the expressiveness of the DST. Later, a family of optimal algorithms working in the general case, i.e. the ones based on the Fast Möbius Transform (FMT) [9], was discovered. Their complexity is $O\left(|\Omega| .2^{|\Omega|}\right)$ in time and $O\left(2^{|\Omega|}\right)$ in space. It has become the de facto standard for the computation of every transformation in DST. Consequently, efforts were made to reduce the size of $\Omega$ to benefit from the optimal algorithms of the FMT. More specifically, [14] refers to the process of conditioning by the combined core (intersection of the unions of all focal sets of each belief source) and lossless coarsening (merging of elements of $\Omega$ which always appear together in focal sets). Also, Monte Carlo methods [14] have been proposed but depend on a number of trials that must be large and grows with $|\Omega|$, in addition to not being exact.

The evidence-based approach concerns all algorithms that aim to reduce the computations to the only subsets that contain information (evidence), called focal sets and usually far less numerous than $2^{|\Omega|}$. This approach, also refered as the obvious one, implicitly originates from the seminal work of Shafer [11] and is often more efficient than the powerset-based one since it only depends on information contained in sources in a quadratic way. Doing so, it allows for the exploitation of the full potential of DST by enabling us to choose any frame of discernment, without concern about its size. Moreover, the evidencebased approach benefits directly from the use of approximation methods, some of which are very efficient [10]. Therefore, this approach seems superior to the FMT in most use cases, above all when $|\Omega|$ is large, where an algorithm with exponential complexity is just intractable.

It is also possible to easily find evidence-based algorithms computing all DST transformation, except for the conjunctive and disjunctive decompositions for which we recently proposed a method [4].

However, since these algorithms rely only on the information contained in sources, they do not exploit the structure of the powerset to reduce the com- 
plexity, leading to situations in which the FMT can be more efficient if almost every subset contains information, i.e. if the number of focal sets tends towards $2^{|\Omega|}[14]$, all the most when no approximation method is employed.

In this paper, we fuse these two approaches into one, proposing new sequences of graphs, in the same fashion as the FMT, that are always more efficient than the FMT and can in addition benefit from evidence-based optimizations. We call them the Efficient Möbius Transformations (EMT). More generally, our approach applies to any function defined on a finite distributive lattice.

Outside the scope of DST, [2] is related to our approach in the sense that we both try to remove redundancy in the computation of the zeta and Möbius transforms on the subset lattice $2^{\Omega}$. However, they only consider the redundancy of computing the image of a subset that is known to be null beforehand. To do so, they only visit sets that are accessible from the focal sets of lowest rank by successive unions with each element of $\Omega$. Here, we demonstrate that it is possible to avoid far more computations by reducing them to specific sets so that each image is only computed once. These sets are the focal points described in [4]. The study of their properties will be carried out in depth in an upcoming article [5]. Besides, our method is more general since it applies to any finite distributive lattice.

Furthermore, an important result of our work resides in the optimal computation of the zeta and Möbius transforms in any intersection-closed family $F$ of sets from $2^{\Omega}$, i.e. with a complexity $O(|\Omega| \cdot|F|)$. Indeed, in the work of [3] on the optimal computation of these transforms in any finite lattice $L$, they embedded $L$ into the Boolean lattice $2^{\Omega}$, obtaining an intersection-closed family $F$ as its equivalent, and found a meta-procedure building a circuit of size $O(|\Omega| .|F|)$ computing the zeta and Möbius transforms. However, they did not managed to build this circuit in less than $O\left(|\Omega| .2^{|\Omega|}\right)$. Given $F$, our Theorem 2 in this paper directly computes this circuit in $O(|\Omega| .|F|)$, while being much simpler.

This paper is organized as follows: Section 2 will present the elements on which our method is built. Section 3 will present our EMT. Section 4 will discuss their complexity and their usage in DST. Finally, we will conclude this article with section 5 .

\section{Background of our method}

Let $(P, \leq)$ be a finite ${ }^{1}$ set partially ordered by $\leq$.

Zeta transform The zeta transform $g: P \rightarrow \mathbb{R}$ of a function $f: P \rightarrow \mathbb{R}$ is defined as follows:

$$
\forall y \in P, \quad g(y)=\sum_{x \leq y} f(x)
$$

\footnotetext{
1 The following definitions hold for lower semifinite partially ordered sets as well, i.e. partially ordered sets such that the number of elements of $P$ lower in the sense of $\leq$ than another element of $P$ is finite. But for the sake of simplicity, we will only talk of finite partially ordered sets.
} 
For example, the commonality function $q$ (resp. the implicability function $b$ ) in DST is the zeta transform of the mass function $m$ for $\left(2^{\Omega}, \supseteq\right)\left(\right.$ resp. $\left(2^{\Omega}, \subseteq\right)$ ).

Möbius transform The Möbius transform of $g$ is $f$. It is defined as follows:

$$
\forall y \in P, \quad f(y)=\sum_{x \leq y} g(x) \cdot \mu(x, y)
$$

where $\mu$ is the Möbius function of $P$.

There is also a multiplicative version with the same properties in which the sum is replaced by a product. An example of this version would be the inverse of the conjunctive (resp. disjunctive) weight function in DST which is the multiplicative Möbius transform of the commonality (resp. implicability) function.

\subsection{Sequence of graphs and computation of the zeta transform}

Consider a procedure $\mathfrak{A}:\left(\mathbb{R}^{P}, \mathcal{G}_{P, \leq},\{+,-, \cdot, /\}\right) \rightarrow \mathbb{R}^{P}$, where $\mathbb{R}^{P}$ is the set of functions of domain $P$ and range $\mathbb{R}$, and $\mathcal{G}_{P, \leq}$ is the set of acyclic directed graphs in which every node is in $P$ and every arrow is a pair $(x, y) \in P^{2}$ such that $x \leq y$. For any such function $m$ and graph $G$, the procedure $\mathfrak{A}(m, G,+)$ outputs a function $z$ such that, for every $y \in P, z(y)$ is the sum of every $m(x)$ where $(x, y)$ is an arrow of $G$. We define its reverse procedure as $\mathfrak{A}(z, G,-)$, which outputs the function $m^{\prime}$ such that, for every $y \in P, m^{\prime}(y)$ is the sum, for every arrow $(x, y)$ of $G$, of $z(x)$ if $x=y$, and $-z(x)$ otherwise. If the arrows of $G$ represent all pairs of $P$ ordered by $\leq$, then $\mathfrak{A}(m, G,+)$ computes the zeta transform $z$ of $m$. Note however that $\mathfrak{A}(z, G,-)$ does not output the Möbius transform $m$ of $z$. For that, $G$ has to be broken down into a sequence of subgraphs (e.g. one subgraph per rank of $y$, in order of increasing rank).

Moreover, the upper bound complexity of these procedures, if $G$ represent all pairs of $P$ ordered by $\leq$, is $O\left(|P|^{2}\right)$. Yet, it is known that the optimal upper bound complexity of the computation of the zeta and Möbius transforms if $P$ is a finite lattice is $O\left(\left.\right|^{\vee} \mathcal{I}(P)|\cdot| P \mid\right)$ (see [3]). Thus, a decomposition of these procedures should lead to a lower complexity at least in this case.

For this, Theorem 3 of [9] defines a necessary and sufficient condition to verify that $\mathfrak{A}\left(\mathfrak{A}\left(\ldots\left(\mathfrak{A}\left(m, H_{1},+\right), \ldots\right), H_{k-1},+\right), H_{k},+\right)=\mathfrak{A}\left(m, G_{\leq},+\right)$, where $H_{i}$ is the $i$-th directed acyclic graph of a sequence $H$ of size $k$, and $G_{\leq}=\left\{(x, y) \in P^{2} / x \leq\right.$ $y\}$. For short, it is said in [9] that $H$ computes the Möbius transformation of $G_{\leq}$. Here, in order to dissipate any confusion, we will say instead that $H$ computes the zeta transformation of $G_{\leq}$.

It is stated in our terms as follows: $H$ computes the zeta transformation of $G_{\leq}$if and only if every arrow from each $H_{i}$ is in $G_{\leq}$and every arrow $g$ from $G_{\leq}$ can be decomposed as a unique path $\left(g_{1}, g_{2}, \ldots, g_{|H|}\right) \in H_{1} \times H_{2} \times \cdots \times H_{|H|}$, i.e. such that the tail of $g$ is the one of $g_{1}$, the head of $g$ is the one of $g_{|H|}$, and $\forall i \in\{1, \ldots,|H|-1\}$, the head of $g_{i}$ is the tail of $g_{i+1}$. 




Fig. 1. Illustration representing the arrows contained in the sequence $H$ computing the zeta transformation of $G_{\subseteq}=\left\{(X, Y) \in 2^{\Omega} \times 2^{\Omega} / X \subseteq Y\right\}$, where $\Omega=\{a, b, c\}$. For the sake of clarity, identity arrows are not displayed. This representation is derived from the one used in [9].

Application to the Boolean lattice $2^{\Omega}$ (FMT) Let $\Omega=\left\{\omega_{1}, \omega_{2}, \ldots, \omega_{n}\right\}$. The sequence $H$ of graphs $H_{i}$ computes the zeta transformation of $G_{\subseteq}=$ $\left\{(X, Y) \in 2^{\Omega} \times 2^{\Omega} / X \subseteq Y\right\}$ if:

$$
H_{i}=\left\{(X, Y) \in 2^{\Omega} \times 2^{\Omega} / Y=X \text { or } Y=X \cup\left\{\omega_{i}\right\}\right\},
$$

where $i \in\{1, \ldots, n\}$. Fig. 1 illustrates the sequence $H$.

Dually, the sequence $\bar{H}$ of graphs $\bar{H}_{i}$ computes the zeta transformation of $G_{\supseteq}=\left\{(X, Y) \in 2^{\Omega} \times 2^{\Omega} / X \supseteq Y\right\}$ if:

$$
\bar{H}_{i}=\left\{(X, Y) \in 2^{\Omega} \times 2^{\Omega} / X=Y \text { or } X=Y \cup\left\{\omega_{i}\right\}\right\} .
$$

The sequences of graphs $H$ and $\bar{H}$ are the foundation of the FMT algorithms. Their execution is $O\left(n .2^{n}\right)$ in time and $O\left(2^{n}\right)$ in space.

\subsection{Sequence of graphs and computation of the Möbius transform}

Now, consider that we have a sequence $H$ computing the zeta transformation of $G_{\leq}$. It easy to see that the procedure $\mathfrak{A}\left(\ldots\left(\mathfrak{A}\left(\mathfrak{A}\left(z, H_{k},-\right), H_{k-1},-\right), \ldots\right), H_{1},-\right)$ deconstructs $z=\mathfrak{A}\left(\mathfrak{A}\left(\ldots\left(\mathfrak{A}\left(m, H_{1},+\right), \ldots\right), H_{k-1},+\right), H_{k},+\right)$, revisiting every arrow in $H$, as required to compute the Möbius transformation. But, to actually compute the Möbius transformation and get $m$ back with $H$ and $\mathfrak{A}$, we have to make sure that the images of $z$ that we add through $\mathfrak{A}$ do not bear redundancies (e.g. if $H$ is the sequence that only contains $G_{\leq}$, then $H$ does compute the Möbius transformation of $G_{\leq}$with Eq. 1, but not with $\mathfrak{A}$ ). For this, we only have to check that for each arrow $(x, y)$ in $G_{\leq}$, there exists at most one path $\left(g_{1}, \ldots, g_{p}\right) \in H_{i_{1}} \times \cdots \times H_{i_{p}}$ where $p \in \mathbb{N}^{*}$ and $\forall j \in\{1, \ldots, p-1\}, 1 \leq i_{j} \leq$ $i_{j+1} \leq i_{j}+1 \leq|H|$ and either $\operatorname{tail}\left(g_{j}\right) \neq \operatorname{head}\left(g_{j}\right)$ or $i_{j-1}<i_{j}<i_{j+1}$ (i.e. which moves right or down in Fig. 1). With this, we know that we do not subtract two images $z_{1}$ and $z_{2}$ to a same $z_{3}$ if one of $z_{1}$ and $z_{2}$ is supposed to be subtracted from the other beforehand. In the end, it is easy to see that, if for each graph $H_{i}$, all element $y \in P$ such that $(x, y) \in H_{i}$ and $\left(y, y^{\prime}\right) \in H_{i}$ where $x \neq y$ verifies $y^{\prime}=y$ (i.e. no "horizontal" path of more than one arrow in each $H_{i}$ ), then the condition is already satisfied by the one of section 2.1. So, if this condition is satisfied, we will say that $H$ computes the Möbius transformation of $G_{\leq}$. 
Application to the Boolean lattice $2^{\Omega}$ (FMT) Resuming the application of section 2.1, for all $X \in 2^{\Omega}$, if $\omega_{i} \notin X$, then there is an arrow $(X, Y)$ in $H_{i}$ where $Y=X \cup\left\{\omega_{i}\right\}$ and $X \neq Y$, but then for any set $Y^{\prime}$ such that $\left(Y, Y^{\prime}\right) \in H_{i}$, we have $Y^{\prime}=Y \cup\left\{\omega_{i}\right\}=Y$. Conversely, if $\omega_{i} \in X$, then the arrow $\left(X, X \cup\left\{\omega_{i}\right\}\right)$ is in $H_{i}$, but its head and tail are equal. Thus, $H$ also computes the Möbius transformation of $G_{\subseteq}$.

\section{$2.3 \quad$ Order theory}

Irreducible elements We note ${ }^{\vee} \mathcal{I}(P)$ the set of join-irreducible elements of $P$, i.e. the elements $i$ such that $i \neq \bigwedge P$ for which it holds that $\forall x, y \in P$, if $x<i$ and $y<i$, then $x \vee y<i$. Dually, we note ${ }^{\wedge} \mathcal{I}(P)$ the set of meet-irreducible elements of $P$, i.e. the elements $i$ such that $i \neq \vee P$ for which it holds that $\forall x, y \in P$, if $x>i$ and $y>i$, then $x \wedge y>i$. For example, in the Boolean lattice $2^{\Omega}$, the join-irreducible elements are the singletons $\{\omega\}$, where $\omega \in \Omega$.

If $P$ is a finite lattice, then every element of $P$ is the join of join-irreducible elements and the meet of meet-irreducible elements.

Support of a function in $P$ The support $\operatorname{supp}(f)$ of a function $f: P \rightarrow \mathbb{R}$ is defined as $\operatorname{supp}(f)=\{x \in P / f(x) \neq 0\}$.

For example, in DST, the set of focal elements of a mass function $m$ is $\operatorname{supp}(m)$.

\subsection{Focal points}

For any function $f: P \rightarrow \mathbb{R}$, we note ${ }^{\wedge} \operatorname{supp}(f)\left(\right.$ resp. $\left.{ }^{\vee} \operatorname{supp}(f)\right)$ the smallest meet-closed (resp. join-closed) subset of $P$ containing $\operatorname{supp}(f)$, i.e.:

$$
\begin{aligned}
& \wedge_{\operatorname{supp}}(f)=\left\{x / \exists S \subseteq \operatorname{supp}(f), S \neq \emptyset, x=\bigwedge_{s \in S} s\right\} \\
& \vee_{\operatorname{supp}(f)} s\left\{x / \exists S \subseteq \operatorname{supp}(f), S \neq \emptyset, x=\bigvee_{s \in S} s\right\}
\end{aligned}
$$

The set of focal points $\stackrel{\circ}{\mathcal{F}}$ of a mass function $m$ from [4] for the conjunctive weight function is ${ }^{\wedge} \operatorname{supp}(m)$. For the disjunctive one, it is ${ }^{\vee} \operatorname{supp}(m)$.

It has been proven in [4] that the image of $2^{\Omega}$ through the conjunctive weight function can be computed without redundancies by only considering the focal points $\wedge^{\wedge} \operatorname{supp}(m)$ in the definition of the multiplicative Möbius transform of the commonality function. The image of all set in $2^{\Omega} \backslash \wedge \operatorname{supp}(m)$ through the conjunctive weight function is 1 . The same can be stated for the disjunctive weight function regarding the implicability function and ${ }^{\vee} \operatorname{supp}(m)$. In the same way, the image of any set in $2^{\Omega} \backslash \wedge \operatorname{supp}(m)$ through the commonality function is only a duplicate of the image of a set in ${ }^{\wedge} \operatorname{supp}(m)$ and can be recovered by searching for its smallest superset in ${ }^{\wedge} \operatorname{supp}(m)$. In fact, as generalized in an upcoming article [5], for any function $f: P \rightarrow \mathbb{R}, \wedge^{\wedge} \operatorname{supp}(f)$ is sufficient to define 
its zeta and Möbius transforms based on the partial order $\geq$, and ${ }^{\vee} \operatorname{supp}(f)$ is sufficient to define its zeta and Möbius transforms based on the partial order $\leq$.

However, considering the case where $P$ is a finite lattice, naive algorithms that only consider ${ }^{\wedge} \operatorname{supp}(f)$ or ${ }^{\vee} \operatorname{supp}(f)$ have upper bound complexities in $O\left(|\wedge \operatorname{supp}(f)|^{2}\right)$ or $O\left(\left|\vee^{\vee} \operatorname{supp}(f)\right|^{2}\right)$, which may be worse than the optimal complexity $O\left(\left.\right|^{\vee} \mathcal{I}(P)|| P \mid.\right)$ for a procedure that considers the whole lattice $P$. In this paper, we propose algorithms with complexities always less than $O(|\vee \mathcal{I}(P)| .|P|)$ computing the image of a meet-closed (e.g. $\left.{ }^{\wedge} \operatorname{supp}(f)\right)$ or join-closed (e.g. $\left.\vee_{\text {supp }}(f)\right)$ subset of $P$ through the zeta or Möbius transform, provided that $P$ is a finite distributive lattice.

\section{Our Efficient Möbius Transformations}

In this section, we consider a function $f: P \rightarrow \mathbb{R}$ where $P$ is a finite distributive lattice (e.g. the Boolean lattice $2^{\Omega}$ ). We present here our Efficient Möbius Transformations as Theorems 1 and 2. The first one describes a way of computing the zeta and Möbius transforms of a function based on the smallest sublattice $\mathcal{L}_{\text {Supp }}(f)$ of $P$ containing both ${ }^{\wedge} \operatorname{supp}(f)$ and ${ }^{\vee} \operatorname{supp}(f)$, which is defined in Proposition 2. The second one goes beyond this optimization by computing these transforms based only on ${ }^{\wedge} \operatorname{supp}(f)$ or ${ }^{\vee} \operatorname{supp}(f)$. Nevertheless, this second approach requires the direct computation of ${ }^{\wedge} \operatorname{supp}(f)$ or ${ }^{\vee} \operatorname{supp}(f)$, which has an upper bound complexity of $O\left(\left.|\operatorname{supp}(f)| \cdot\right|^{\wedge} \operatorname{supp}(f) \mid\right)$ or $O\left(\left.|\operatorname{supp}(f)| \cdot\right|^{\vee} \operatorname{supp}(f) \mid\right)$, which may be more than $O\left(\left.\right|^{\vee} \mathcal{I}(P)|| P \mid.\right)$ if $|\operatorname{supp}(f)| \gg|\vee \mathcal{I}(P)|$.

Lemma 1 (Safe join). Let us consider a finite distributive lattice L. For all $i \in{ }^{\vee} \mathcal{I}(L)$ and for all $x, y \in L$ such that $i \not \leq x$ and $i \not \leq y$, we have $i \not \leq x \vee y$.

Proof. By definition of a join-irreducible element, we know that $\forall i \in{ }^{\vee} \mathcal{I}(L)$ and for all $a, b \in L$, if $a<i$ and $b<i$, then $a \vee b<i$. Moreover, for all $x, y \in L$ such that $i \not \leq x$ and $i \not \leq y$, we have equivalently $i \wedge x<i$ and $i \wedge y<i$. Thus, we get that $(i \wedge x) \vee(i \wedge y)<i$. Since $L$ satisfies the distributive law, this implies that $(i \wedge x) \vee(i \wedge y)=i \wedge(x \vee y)<i$, which means that $i \not \leq x \vee y$.

Proposition 1 (Iota elements of subsets of $P$ ). For any $S \subseteq P$, the joinirreducible elements of the smallest sublattice $L_{S}$ of $P$ containing $S$ are:

$$
\iota(S)=\left\{\bigwedge\{s \in S / s \geq i\} / i \in{ }^{\vee} \mathcal{I}(P) \text { and } \exists s \in S, \quad s \geq i\right\} .
$$

Proof. First, it can be easily shown that the meet of any two elements of $\iota(S)$ is either $\bigwedge S$ or in $\iota(S)$. Then, suppose that we generate $L_{S}$ with the join of elements of $\iota(S)$, to which we add the element $\bigwedge S$. Then, since $P$ is distributive, we have that for all $x, y \in L_{S}$, their meet $x \wedge y$ is either $\wedge S$ or equal to the join of every meet of pairs $\left(i_{S, x}, i_{S, y}\right) \in \iota(S)^{2}$, where $i_{S, x} \leq x$ and $i_{S, y} \leq y$. Thus, $x \wedge y \in L_{S}$, which implies that $L_{S}$ is a sublattice of $P$. In addition, notice that for each nonzero element $s \in S$ and for all $i \in{ }^{\vee} \mathcal{I}(P)$ such that $s \geq i$, we also have by construction $s \geq i_{S} \geq i$, where $i_{S}=\bigwedge\{s \in S / s \geq i\}$. Therefore, we 
have $s=\bigvee\left\{i \in{ }^{\vee} \mathcal{I}(P) / s \geq i\right\}=\bigvee\{i \in \iota(S) / s \geq i\}$, i.e. $s \in L_{S}$. Besides, if $\bigwedge P \in S$, then it is equal to $\bigwedge S$, which is also in $L_{S}$ by construction. So, $S \subseteq L_{S}$. It follows that the meet or join of every nonempty subset of $S$ is in $L_{S}$, i.e. $M_{S} \subseteq L_{S}$ and $J_{S} \subseteq L_{S}$, where $M_{S}$ is the smallest meet-closed subset of $P$ containing $S$ and $J_{S}$ is the smallest join-closed subset of $P$ containing $S$. Furthermore, $\iota(S) \subseteq M_{S}$ which means that we cannot build a smaller sublattice of $P$ containing $S$. Therefore, $L_{S}$ is the smallest sublattice of $P$ containing $S$.

Finally, for any $i \in{ }^{\vee} \mathcal{I}(P)$ such that $\exists s \in S, s \geq i$, we note $i_{S}=\bigwedge\{s \in$ $S / s \geq i\}$. For all $x, y \in L_{S}$, if $i_{S}>x$ and $i_{S}>y$, then by construction of $\iota(S)$, we have $i \not \leq x$ and $i \not \leq y$ (otherwise, $i_{S}$ would be less than $x$ or $y$ ), which implies by Lemma 1 that $i \not \leq x \vee y$. Since $i \leq i_{S}$, we have necessarily $i_{S}>x \vee y$. Therefore, $i_{S}$ is a join-irreducible element of $L_{S}$.

Proposition 2 (Lattice support). The smallest sublattice of $P$ containing both ${ }^{\wedge} \operatorname{supp}(f)$ and ${ }^{\vee} \operatorname{supp}(f)$, noted ${ }^{\mathcal{L}} \operatorname{supp}(f)$, can be defined as:

$$
\mathcal{L}_{\operatorname{supp}(f)}=\{\bigvee X / X \subseteq \iota(\operatorname{supp}(f)), X \neq \emptyset\} \cup\{\bigwedge \operatorname{supp}(f)\} .
$$

More specifically, ${ }^{\vee}$ supp $(f)$ is contained in the upper closure ${ }^{\mathcal{L}, \uparrow}$ supp $(f)$ of $\operatorname{supp}(f)$ in $\mathcal{L}_{\text {supp }}(f)$ :

$$
{ }^{\mathcal{L}, \uparrow} \operatorname{supp}(f)=\left\{x \in \mathcal{L}^{\operatorname{supp}}(f) / \exists s \in \operatorname{supp}(f), s \leq x\right\},
$$

and $^{\wedge} \operatorname{supp}(f)$ is contained in the lower closure ${ }^{\mathcal{L}, \downarrow} \operatorname{supp}(f)$ of $\operatorname{supp}(f)$ in $\mathcal{L}_{\text {supp }}(f)$ :

$$
\mathcal{L}, \downarrow \operatorname{supp}(f)=\left\{x \in \mathcal{L}^{\operatorname{supp}}(f) / \exists s \in \operatorname{supp}(f), s \geq x\right\} .
$$

These sets can be computed in less than respectively $O(|\iota(\operatorname{supp}(f))| \cdot|\mathcal{L}, \uparrow \operatorname{supp}(f)|)$ and $O\left(\left.|\iota(\operatorname{supp}(f))| \cdot\right|^{\mathcal{L}, \downarrow} \operatorname{supp}(f) \mid\right)$, which is at most $O\left(\left.\right|^{\vee} \mathcal{I}(P)|| P \mid.\right)$.

Proof. The proof is immediate here, considering Proposition 1 and its proof. In addition, since ${ }^{\wedge} \operatorname{supp}(f)$ only contains the meet of elements of supp $(f)$, all element of ${ }^{\wedge} \operatorname{supp}(f)$ is less than at least one element of supp $(f)$. Similarly, since $\vee_{\text {supp }}(f)$ only contains the join of elements of $\operatorname{supp}(f)$, all element of $\vee \operatorname{supp}(f)$ is greater than at least one element of supp $(f)$. Hence ${ }^{\mathcal{L}, \downarrow} \operatorname{supp}(f)$ and ${ }^{\mathcal{L}, \uparrow} \operatorname{supp}(f)$.

As pointed out in [8], a special ordering of the join-irreducible elements of a lattice when using the Fast Zeta Transform [3] leads to the optimal computation of its zeta and Möbius transforms. Here, we use this ordering to build our EMT for finite distributive lattices in a way similar to [8] but without the need to check the equality of the decompositions into the first $j$ join-irreducible elements at each step.

Corollary 1 (Join-irreducible ordering). Let us consider a finite distributive lattice $L$ and let its join-irreducible elements ${ }^{\vee} \mathcal{I}(L)$ be ordered such that $\forall i_{k}, i_{l} \in{ }^{\vee} \mathcal{I}(L), k<l \Rightarrow i_{k} \geq i_{l}$. We note ${ }^{\vee} \mathcal{I}(L)_{k}=\left\{i_{1}, \ldots, i_{k-1}, i_{k}\right\}$.

For all element $i_{k} \in{ }^{\vee} \mathcal{I}(L)$, we have $i_{k} \not \leq \bigvee^{\vee} \mathcal{I}(L)_{k-1}$.

If $L$ is a graded lattice (i.e. a lattice equipped with a rank function $\rho: L \rightarrow \mathbb{N}$ ), then $\rho\left(i_{1}\right) \leq \rho\left(i_{2}\right) \leq \cdots \leq \rho\left(i_{|\vee \mathcal{I}(L)|}\right)$ implies this ordering. For example, in DST, $P=2^{\Omega}$, so for all $A \in P, \rho(A)=|A|$. 




Fig. 2. Illustration representing the arrows contained in the sequence $H$ when computing the zeta transformation of $G \subseteq=\left\{(x, y) \in L^{2} / x \subseteq y\right\}$, where $L=$ $\{\emptyset,\{a\},\{d\},\{a, d\},\{c, d, f\},\{a, c, d, f\}, \Omega\}$ with $\Omega=\{a, b, c, d, e, f\}$ and ${ }^{\vee} \mathcal{I}(L)=$ $\{\{a\},\{d\},\{c, d, f\}, \Omega\}$. For the sake of clarity, identity arrows are not displayed.

Proof. Since the join-irreducible elements are ordered such that $\forall i_{k}, i_{l} \in{ }^{\vee} \mathcal{I}(L)$, $k<l \Rightarrow i_{k} \nsupseteq i_{l}$, it is trivial to see that for any $i_{l} \in{ }^{\vee} \mathcal{I}(L)$ and $i_{k} \in{ }^{\vee} \mathcal{I}(L)_{l-1}$, we have $i_{k} \nsucceq i_{l}$. Then, using Lemma 1 by recurrence, it is easy to get that $i_{l} \not \leq \bigvee^{\vee} \mathcal{I}(L)_{l-1}$.

Theorem 1 (Efficient Möbius Transformation in a distributive lattice). Let us consider a finite distributive lattice $L$ (such as $\left.\mathcal{L}_{\text {supp }}(f)\right)$ and let its join-irreducible elements ${ }^{\vee} \mathcal{I}(L)$ be ordered such that $\forall i_{k}, i_{l} \in{ }^{\vee} \mathcal{I}(L)$, $k<l \Rightarrow i_{k} \nsupseteq i_{l}$. We note $n=\left.\right|^{\vee} \mathcal{I}(L) \mid$.

The sequence $H$ of graphs $H_{k}$ computes the zeta and Möbius transformations of $G_{\leq}=\left\{(x, y) \in L^{2} / x \leq y\right\}$ if:

$$
H_{k}=\left\{(x, y) \in L^{2} / y=x \text { or } y=x \vee i_{\bar{k}}\right\},
$$

where $\bar{k}=n+1-k$. This sequence is illustrated in Fig. 2. Its execution is $O(n .|L|)$.

Dually, the sequence $\bar{H}$ of graphs $\bar{H}_{k}$ computes the zeta and Möbius transformations of $G_{\geq}=\left\{(x, y) \in L^{2} / x \geq y\right\}$ if:

$$
\bar{H}_{k}=\left\{(x, y) \in L^{2} / x=y \text { or } x=y \vee i_{k}\right\} .
$$

Proof. By definition, for all $k$ and $\forall(x, y) \in H_{k}$, we have $x, y \in L$ and $x \leq y$, i.e. $(x, y) \in G_{\leq}$. Reciprocally, $\forall(x, y) \in G_{\leq}$, we have $x \leq y$, which can be decomposed as a unique path $\left(g_{1}, g_{2}, \ldots, g_{n}\right) \in H_{1} \times H_{2} \times \cdots \times H_{n}$ :

Similarly to the FMT, the sequence $H$ builds unique paths simply by generating the whole lattice step by step with each join-irreducible element of $L$. However, unlike the FMT, the join-irreducible elements of $L$ are not necessarily atoms. Doing so, pairs of join-irreducible elements may be ordered, causing the sequence $H$ to skip or double some elements. And even if all the join-irreducible elements of $L$ are atoms, since $L$ is not necessarily a Boolean lattice, the join of two atoms may be greater than a third atom (e.g. if $L$ is the diamond lattice), 
leading to the same issue. Indeed, to build a unique path between two elements $x, y$ of $L$ such that $x \leq y$, we start from $x$. Then at step 1 , we get to the join $x \vee i_{n}$ if $i_{n} \leq y$ (we stay at $x$ otherwise, i.e. identity arrow), then we get to $x \vee i_{n} \vee i_{n-1}$ if $i_{n-1} \leq y$, and so on until we get to $y$. However, if we have $i_{n} \leq x \vee i_{n-1}$, with $i_{n} \not \leq x$, then there are at least two paths from $x$ to $y$ : one passing by the join with $i_{n}$ at step 1 and one passing by the identity arrow instead.

More generally, this kind of issue may only appear if there is a $k$ where $i_{k} \leq x \vee \bigvee^{\vee} \mathcal{I}(L)_{k-1}$ with $i_{k} \not \leq x$, where ${ }^{\vee} \mathcal{I}(L)_{k-1}=\left\{i_{k-1}, i_{k-2}, \ldots, i_{1}\right\}$. But, since $L$ is a finite distributive lattice, and since its join-irreducible elements are ordered such that $\forall i_{j}, i_{l} \in{ }^{\vee} \mathcal{I}(L), j<l \Rightarrow i_{j} \nsupseteq i_{l}$, we have by Corollary 1 that $i_{k} \not \leq \bigvee^{\vee} \mathcal{I}(L)_{k-1}$. So, if $i_{k} \not \leq x$, then by Lemma 1, we also have $i_{k} \not{Z}$ $x \vee \bigvee^{\vee} \mathcal{I}(L)_{k-1}$. Thereby, there is a unique path from $x$ to $y$, meaning that the condition of section 2.1 is satisfied. $H$ computes the zeta transformation of $G_{\leq}$.

Also, $\forall x \in L$, if $i_{k} \not \leq x$, then there is an arrow $(x, y)$ in $H_{k}$ where $y=x \vee \bar{i}_{k}$ and $x \neq y$, but then for any element $y^{\prime}$ such that $\left(y, y^{\prime}\right) \in H_{k}$, we have $y^{\prime}=$ $y \vee i_{k}=y$. Conversely, if $i_{k} \leq x$, then the arrow $\left(x, x \vee i_{k}\right)$ is in $H_{k}$, but its head and tail are equal. Thus, the condition of section 2.2 is satisfied. $H$ also computes the Möbius transformation of $G_{<}$.

Finally, to obtain $\bar{H}$, we only need to reverse the paths of $H$, i.e. reverse the arrows in each $H_{k}$ and reverse the sequence of join-irreducible elements.

The procedure described in Theorem 1 to compute the zeta and Möbius transforms of a function on $P$ is always less than $O\left(\left.\right|^{\vee} \mathcal{I}(P)|\cdot| P \mid\right)$. Its upper bound complexity for the distributive lattice $L={ }^{\mathcal{L}} \operatorname{supp}(f)$ is $O(|\vee \mathcal{I}(L)| .|L|)$, which is actually the optimal one for a lattice.

Yet, we can reduce this complexity even further if we have ${ }^{\wedge} \operatorname{supp}(f)$ or $\vee \operatorname{supp}(f)$. This is the motivation behind the procedure decribed in the following Theorem 2. As a matter of fact, [3] proposed a meta-procedure producing an algorithm that computes the zeta and Möbius transforms in an arbitrary intersection-closed family $F$ of sets of $2^{\Omega}$ with a circuit of size $O(|\Omega| .|F|)$. However, this meta-procedure is $O\left(|\Omega| .2^{|\Omega|}\right)$. Here, Theorem 2 provides a procedure that directly computes the zeta and Möbius transforms with the optimal complexity $O(|\Omega| .|F|)$, while being much simpler. Besides, our method is far more general since it has the potential (depending on data structure) to reach this complexity in any meet-closed subset of a finite distributive lattice.

Theorem 2 (Efficient Möbius Transformation in a join-closed or meetclosed subset of $P$ ). Let us consider a meet-closed subset $M$ of $P$ (such ${ }_{\text {as }} \wedge$ supp $\left.(f)\right)$. Also, let the join-irreducible elements $\iota(M)$ be ordered such that $\forall i_{k}, i_{l} \in \iota(M), k<l \Rightarrow i_{k} \nsupseteq i_{l}$.

The sequence $H^{M}$ of graphs $H_{k}^{M}$ computes the zeta and Möbius transformations of $G_{\geq}^{M}=\left\{(x, y) \in M^{2} / x \geq y\right\}$ if:

$$
\begin{aligned}
H_{k}^{M}=\left\{(x, y) \in M^{2} / x=y\right. & \\
& \text { or } \left.\left(x=\bigwedge\left\{s \in M / s \geq y \vee i_{k}\right\} \text { and } y \vee \bigvee \iota(M)_{k} \geq x\right)\right\},
\end{aligned}
$$




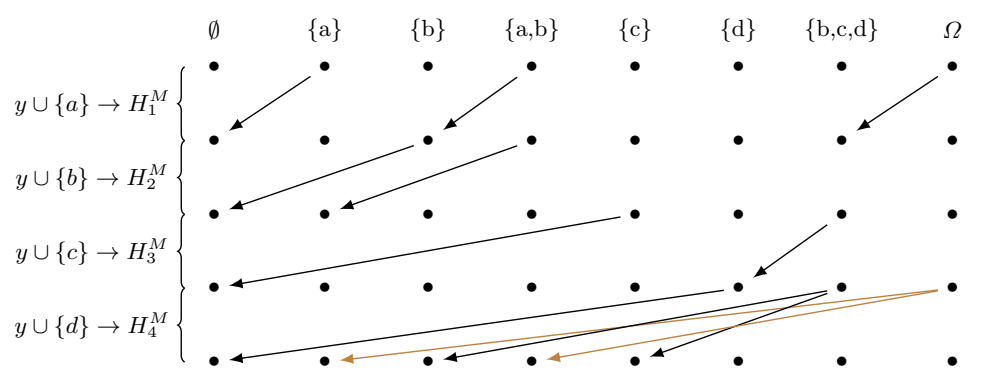

Fig. 3. Illustration representing the arrows contained in the sequence $H^{M}$ when computing the zeta transformation of $G_{\supseteq}^{M}=\left\{(x, y) \in M^{2} / x \supseteq y\right\}$, where $M=\{\emptyset,\{a\},\{b\},\{a, b\},\{c\},\{d\},\{b, c, d\}, \Omega\}$ with $\Omega=\{a, b, c, d\}$ and $\iota(M)=$ $\{\{a\},\{b\},\{c\},\{d\}\}$. For the sake of clarity, identity arrows are not displayed.

where $\iota(M)_{k}=\left\{i_{1}, i_{2}, \ldots, i_{k}\right\}$. This sequence is illustrated in Fig. 3. Its execution is $O(|\iota(M)| .|M| . \epsilon)$, where $\epsilon$ represents the number of operations required to obtain the proxy element $\bigwedge\left\{s \in M / s \geq y \vee i_{k}\right\}$ of $x$. It can be as low as 1 operation ${ }^{2}$.

Dually, the expression of $\overline{H^{M}}$ follows the same pattern, simply reversing the paths of $H^{M}$ by reversing the arrows in each $H_{k}^{M}$ and reversing the sequence of join-irreducible elements.

Similarly, if $P$ is a Boolean lattice, then the dual $H^{J}$ of this sequence $H^{M}$ of graphs computes the zeta and Möbius transformations of $G_{\leq}^{J}=\{(x, y) \in$ $\left.J^{2} / x \leq y\right\}$, where $J$ is a join-closed subset of $P\left(\right.$ such as ${ }^{\vee}$ supp $\left.(f)\right)$. Let the meet-irreducible elements $\bar{\imath}(J)$ of the smallest sublattice of $P$ containing $J$ be ordered such that $\forall \bar{i}_{k}, \bar{i}_{l} \in \bar{\iota}(J), k<l \Rightarrow \bar{i}_{k} \not \leq \bar{i}_{l}$. We have:

$$
\begin{aligned}
H_{k}^{J}=\left\{(x, y) \in J^{2} / x=y\right. & \\
& \left.\quad \text { or }\left(x=\bigvee\left\{s \in J / s \leq y \wedge \bar{i}_{k}\right\} \text { and } y \wedge \bigwedge \bar{\iota}(J)_{k} \leq x\right)\right\},
\end{aligned}
$$

where $\bar{\iota}(J)_{k}=\left\{\bar{i}_{1}, \bar{i}_{2}, \ldots, \bar{i}_{k}\right\}$.

Dually, the expression of $\overline{H^{J}}$ follows the same pattern, simply reversing the paths of $H^{J}$ by reversing the arrows in each $H_{k}^{J}$ and reversing the sequence of meet-irreducible elements.

Proof. By definition, for all $k$ and $\forall(x, y) \in H_{k}^{M}$, we have $x, y \in M$ and $x \geq y$, i.e. $(x, y) \in G_{\geq}^{M}$. Reciprocally, $\forall(x, y) \in G_{\geq}^{M}$, we have $x \geq y$, which can be decomposed as a unique path $\left(g_{1}, g_{2}, \ldots, g_{|\iota(M)|}\right) \in H_{1}^{M} \times H_{2}^{M} \times \cdots \times H_{|\iota(M)|}^{M}$ :

The idea is that we use the same procedure as in Theorem 1 that builds unique paths simply by generating all elements of a finite distributive lattice $L$ based on

${ }^{2}$ This unit cost can be obtained when $P=2^{\Omega}$ using a dynamic binary tree as data structure for the representation of $M$. With it, finding the proxy element only takes the reading of a binary string, considered as one operation. Further details will soon be available in an extended version of this work [5]. 
the join of its join-irreducible elements step by step, as if we had $M \subseteq L$, except that we remove all elements that are not in $M$. Doing so, the only difference $i s$ that the join $y \vee i_{k}$ of an element $y$ of $M$ with a join-irreducible $i_{k} \in \iota(M)$ of this hypothetical lattice $L$ is not necessary in $M$. However, thanks to the meet-closure of $M$ and to the synchronizing condition $y \vee \vee \iota(M)_{k} \geq p$, we can "jump the gap" between two elements $y$ and $p$ of $M$ separated by elements of $L \backslash M$ and maintain the unicity of the path between any two elements $x$ and $y$ of $M$. Indeed, for all join-irreducible element $i_{k} \in \iota(M)$, if $x \geq y \vee i_{k}$, then since $M$ is meet-closed, we have an element $p$ of $M$ that we call proxy such that $p=\bigwedge\left\{s \in M / s \geq y \vee i_{k}\right\}$. Yet, we have to make sure that (1) $p$ can only be obtained from $y$ with exactly one particular $i_{k}$ if $p \neq y$, and (2) that the sequence of these particular join-irreducible elements forming the arrows of the path from $x$ to $y$ are in the correct order. This is the purpose of the synchronizing condition $y \vee \bigvee \iota(M)_{k} \geq p$.

For (1), we will show that for a same proxy $p$, it holds that $\exists ! k \in[1,|\iota(M)|]$ such that $p \neq y, y \vee \bigvee \iota(M)_{k} \geq p$ and $y \nsupseteq i_{k}$. Recall that we ordered the elements $\iota(M)$ such that $\forall i_{j}, i_{l} \in \iota(M), j<l \Rightarrow i_{j} \geq i_{l}$. Let us note $k$ the greatest index among $[1,|\iota(M)|]$ such that $p \geq i_{k}$ and $y \nsupseteq i_{k}$. It is easy to see that the synchonizing condition is statisfied for $i_{k}$. Then, for all $j \in[1, k-1]$, Corollary 1 and Lemma 1 give us that $y \vee \bigvee \iota(M)_{j} \geq i_{k}$, meaning that $y \vee \bigvee \iota(M)_{j} \geq p$. For all $j \in[k+1,|\iota(M)|]$, either $y \geq i_{j}$ (i.e. $p=y \vee i_{j}=y$ ) or $p \nsupseteq i_{j}$. Either way, it is impossible to reach $p$ from $y \vee i_{j}$. Therefore, there exists a unique path from $y$ to $p$ that takes the arrow $(p, y)$ from $H_{k}^{M}$.

Concerning (2), for all $(x, y) \in G_{\geq}^{M}, x \neq y$, let us note the proxy element $p_{1}=\bigwedge\left\{s \in M / s \geq y \vee i_{k_{1}}\right\}$ where $\bar{k}_{1}$ is the greatest index among $[1,|\iota(M)|]$ such that $p_{1} \geq i_{k_{1}}$ and $y \nsupseteq i_{k_{1}}$. We have $\left(p_{1}, y\right) \in H_{k_{1}}^{M}$. Let us suppose that there exists another proxy element $p_{2}$ such that $p_{2} \neq p_{1}, x \geq p_{2}$ and $p_{2}=\bigwedge\{s \in$ $\left.M / s \geq p_{1} \vee i_{k_{2}}\right\}$ where $k_{2}$ is the greatest index among $[1,|\iota(M)|]$ such that $p_{2} \geq i_{k_{2}}$ and $p_{1} \geq i_{k_{2}}$. We have $\left(p_{2}, p_{1}\right) \in H_{k_{2}}^{M}$. Since $p_{2}>p_{1}$ and $p_{1} \geq i_{k_{1}}$, we have that $p_{2} \geq i_{k_{1}}$, i.e. $k_{2} \neq k_{1}$. So, two cases are possible: either $k_{1}>k_{2}$ or $k_{1}<k_{2}$. If $k_{1}>k_{2}$, then there is a path $\left(\left(p_{2}, p_{1}\right),\left(p_{1}, p_{1}\right), \ldots,\left(p_{1}, p_{1}\right),\left(p_{1}, y\right)\right)$ from $p_{2}$ to $y$. Moreover, we know that at step $k_{1}$, we get $p_{1}$ from $y$ and that we have $p_{2} \geq i_{k_{1}}$ and $y \nsupseteq i_{k_{1}}$, meaning that there could only exist an arrow $\left(p_{2}, y\right)$ in $H_{k_{3}}^{M}$ if $k_{3}>k_{1}>k_{2}$. Suppose this $k_{3}$ exists. Then, since $k_{3}>k_{1}>k_{2}$, we have that $p_{2} \geq i_{k_{3}}$ and $y \nsupseteq i_{k_{3}}$, but also $p_{1} \nsupseteq i_{k_{3}}$ since we would have $k_{1}=k_{3}$ otherwise. This implies that $k_{2}=k_{3}$, which is impossible. Therefore, there is no $k_{3}$ such that $\left(p_{2}, y\right) \in H_{k_{3}}^{M}$, i.e. there is a unique path from $p_{2}$ to $y$. Otherwise, if $k_{1}<k_{2}$, then the latter path between $p_{2}$ and $y$ does not exist. But, since $p_{1} \nsucceq i_{k_{2}}$ and $p_{1} \geq y$, we have $y \nsupseteq i_{k_{2}}$, meaning that there exists an arrow $\left(p_{2}, y\right) \in H_{k_{2}}^{M}$, which forms a unique path from $p_{2}$ to $y$. The recurrence of this reasoning enables us to conclude that there is a unique path from $x$ to $y$.

Thus, the condition of section 2.1 is satisfied. $H^{M}$ computes the zeta transformation of $G_{\geq}^{M}$. Also, for the same reasons as with Theorem 1, we have that $H^{M}$ computes the Möbius transformation of $G_{\geq}^{M}$. The proof for $H^{J}$ and $G_{\leq}^{J}$ is analog if $P$ is a Boolean lattice. 


\section{Discussions for Dempster-Shafer Theory}

In DST, we work with $P=2^{\Omega}$, in which the singletons are its join-irreducible elements. If $|\operatorname{supp}(f)|$ is of same order of magnitude as $n$ or lower, where $n=|\Omega|$, then we can compute the focal points ${ }^{\wedge} \operatorname{supp}(f)$ or ${ }^{\vee} \operatorname{supp}(f)$ and use our Efficient Möbius Transformation of Theorem 2 to compute any DST transformation (e.g. the commonality/implicability function, the conjunctive/disjunctive weight function, etc, i.e. wherever the FMT applies) in at most $O(n .|\operatorname{supp}(f)|+$ $\left.\left.|\iota(\operatorname{supp}(f))| \cdot\right|^{R} \operatorname{supp}(f) \mid\right)$ operations, where $R \in\{\wedge, \vee\}$, which is at most $O\left(n .2^{n}\right)$.

Otherwise, we can compute ${ }^{\mathcal{L}, \uparrow} \operatorname{supp}(f)$ or ${ }^{\mathcal{L}, \downarrow} \operatorname{supp}(f)$ of Proposition 2, and then use the Efficient Möbius Transformation of Theorem 1 to compute the same DST transformations in $O\left(n \cdot|\operatorname{supp}(f)|+\left.|\iota(\operatorname{supp}(f))| \cdot\right|^{\mathcal{L}, A} \operatorname{supp}(f) \mid\right)$ operations, where $A \in\{\uparrow, \downarrow\}$, which is at most $O\left(n .2^{n}\right)$.

Therefore, we can always compute DST transformations more efficiently than the FMT with the EMT if $\operatorname{supp}(f)$ is given.

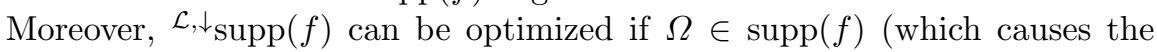
equality $\left.\mathcal{L}, \downarrow_{\operatorname{supp}}(f)=\mathcal{L}_{\operatorname{Supp}}(f)\right)$. Indeed, one can equivalently compute the lattice ${ }^{\mathcal{L}, \downarrow}(\operatorname{supp}(f) \backslash\{\Omega\})$, execute the EMT of Theorem 1, and then add the value on $\Omega$ to the value on all sets of $\mathcal{L}, \downarrow(\operatorname{supp}(f) \backslash\{\Omega\})$. Dually, the same can be done with ${ }^{\mathcal{L}, \uparrow}(\operatorname{supp}(f) \backslash\{\emptyset\})$. This trick can be particularly useful in the case of the conjunctive or disjunctive weight function, which requires that $\operatorname{supp}(f)$ contains respectively $\Omega$ or $\emptyset$.

Also, optimizations built for the FMT, such as the reduction of $\Omega$ to the core $\mathcal{C}$ or its optimal coarsened version $\Omega^{\prime}$, are already encoded in the use of the function $\iota$ (see Example 1), but optimizations built for the evidence-based approach, such as approximations by reduction of the number of focal sets, i.e. reducing the size of $\operatorname{supp}(f)$, can still greatly enhance the EMT.

Finally, while it was proposed in [9] to fuse two mass functions $m_{1}$ and $m_{2}$ using Dempster's rule by computing the corresponding commonality functions $q_{1}$ and $q_{2}$ in $O\left(n .2^{n}\right)$, then $q_{12}=q_{1} . q_{2}$ in $O\left(2^{n}\right)$ and finally computing back the fused mass function $m_{12}$ from $q_{12}$ in $O\left(n .2^{n}\right)$, here we propose an even greater detour that has a lower complexity. Indeed, by computing $q_{1}$ and $q_{2}$ on ${ }^{\wedge} \operatorname{supp}\left(m_{1}\right)$ and ${ }^{\wedge} \operatorname{supp}\left(m_{2}\right)$, then the conjunctive weight functions $w_{1}$ and $w_{2}$ on these same elements, we get $w_{12}=w_{1} \cdot w_{2}$ in $O\left(\left.\right|^{\wedge} \operatorname{supp}\left(m_{1}\right) \cup\right.$

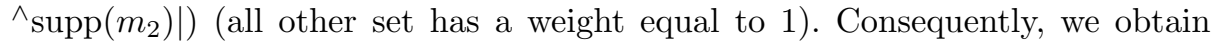
the set $\operatorname{supp}\left(1-w_{12}\right) \subseteq \wedge^{\wedge} \operatorname{supp}\left(m_{1}\right) \cup{ }^{\wedge} \operatorname{supp}\left(m_{2}\right)$ which can be used to com-

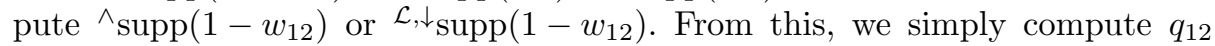
and then $m_{12}$ in $O\left(n \cdot\left|\operatorname{supp}\left(1-w_{12}\right)\right|+\left.\left|\iota\left(\operatorname{supp}\left(1-w_{12}\right)\right)\right| \cdot\right|^{\wedge} \operatorname{supp}\left(1-w_{12}\right) \mid\right)$ or $O\left(n \cdot\left|\operatorname{supp}\left(1-w_{12}\right)\right|+\left.\left|\iota\left(\operatorname{supp}\left(1-w_{12}\right)\right)\right| \cdot\right|^{\mathcal{L}, \downarrow} \operatorname{supp}\left(1-w_{12}\right) \mid\right)$.

Example 1 (Consonant case). If $\operatorname{supp}(f)=\left\{F_{1}, F_{2}, \ldots, F_{K}\right\}$ such that $F_{1} \subset$ $F_{2} \subset \cdots \subset F_{K}$, then the coarsening $\Omega^{\prime}$ of $\Omega$ will have an element for each element of $\operatorname{supp}(f)$, while $\iota(\operatorname{supp}(f))$ will have a set of elements for each element of $\operatorname{supp}(f)$. So, we get $\left|\Omega^{\prime}\right|=|\iota(\operatorname{supp}(f))|=K$. But, $\Omega^{\prime}$ is then used to generate the Boolean lattice $2^{\Omega^{\prime}}$, of size $2^{K}$, where $\iota(\operatorname{supp}(f))$ is used to generate an arbitrary lattice ${ }^{\mathcal{L}} \operatorname{supp}(f)$, of size $K$ in this particular case $(K+1$ if $\emptyset \in \operatorname{supp}(f))$. 


\section{Conclusion}

In this paper, we proposed the Efficient Möbius Transformations (EMT), which are general procedures to compute the zeta and Möbius transforms of any function defined on any finite distributive lattice with optimal complexity. They are based on our reformulation of the Möbius inversion theorem with focal points only, featured in an upcoming detailed article [5] currently in preparation. The EMT optimally exploit the information contained in both the support of this function and the structure of distributive lattices. Doing so, the EMT always perform better than the optimal complexity for an algorithm considering the whole lattice, such as the FMT for all DST transformations, given the support of this function. In [5], we will see that our approach is still more efficient when this support is not given. This forthcoming article will also feature examples of application in DST, algorithms and implementation details.

\section{References}

1. Barnett, J.A.: Computational Methods for a Mathematical Theory of Evidence. Proc. of IJCAI 81, 868-875 (1981)

2. Björklund, A., Husfeldt, T., Kaski, P., Koivisto, M.: Trimmed Moebius inversion and graphs of bounded degree. Theory of Computing Systems 47(3), 637-654 (2010)

3. Björklund, A., Husfeldt, T., Kaski, P., Koivisto, M., Nederlof, J., Parviainen, P.: Fast zeta transforms for lattices with few irreducibles. ACM TALG 12(1), 4 (2016)

4. Chaveroche, M., Davoine, F., Cherfaoui, V.: Calcul exact de faible complexité des décompositions conjonctive et disjonctive pour la fusion d'information. Proc. of GRETSI (2019)

5. Chaveroche, M., Davoine, F., Cherfaoui, V.: Efficient algorithms for Möbius Transformations and their applications to Dempster-Shafer Theory. Manuscript available on request (2019)

6. Dempster, A.: A Generalization of Bayesian Inference. Journal of the Royal Statistical Society. Series B (Methodological) 30 (1968)

7. Gordon, J., Shortliffe, E.H.: A method for managing evidential reasoning in a hierarchical hypothesis space. Artificial intelligence 26(3), 323-357 (1985)

8. Kaski, P., Kohonen, J., Westerbäck, T.: Fast Möbius inversion in semimodular lattices and U-labelable posets. arXiv preprint arXiv:1603.03889 (2016)

9. Kennes, R.: Computational aspects of the Mobius transformation of graphs. IEEE Transactions on Systems, Man, and Cybernetics 22(2), 201-223 (1992)

10. Sarabi-Jamab, A., Araabi, B.N.: Information-Based Evaluation of Approximation Methods in Dempster-Shafer Theory. IJUFKS 24(04), 503-535 (2016)

11. Shafer, G.: A Mathematical Theory of Evidence. Princeton University Press, Princeton (1976)

12. Shafer, G., Logan, R.: Implementing Dempster's rule for hierarchical evidence. Artificial Intelligence 33(3), 271-298 (1987)

13. Shenoy, P.P., Shafer, G.: Propagating Belief Functions with Local Computations. IEEE Expert 1(3), 43-52 (1986)

14. Wilson, N.: Algorithms for Dempster-Shafer Theory. In: Handbook of Defeasible Reasoning and Uncertainty Management Systems: Algorithms for Uncertainty and Defeasible Reasoning, pp. 421-475. Springer Netherlands (2000) 